\title{
Blood Pressure and Mortality: Joint Effect of Blood Pressure Measures
}

\author{
Jung Ki Kim*, Eileen M. Crimmins \\ Davis School of Gerontology, University of Southern California, USA
}

\begin{abstract}
We examine how combinations of systolic and diastolic blood pressure levels and pulse pressure levels predicted mortality risk. Respondents are those aged over 50 from the Health and Retirement Study $(\mathrm{N}=10,366)$ who provided blood pressure measures in 2006/2008. Systolic and diastolic blood pressures were measured three times; and we averaged the three readings. Pulse pressure was calculated as systolic minus diastolic blood pressure. Seven combinations of systolic and diastolic blood pressure (low/normal/high of each) and three levels of pulse pressure (low/normal/high) were used to categorize blood pressure. Over 1 to 10 years of follow-up (average follow-up time of 7.8 years), 2,820 respondents died after blood pressure measurement in 2006/2008. Potential covariates including age, gender, education, BMI, total cholesterol, HbA1c, antihypertensive medication intake and lifetime-smoking pack years were adjusted in Cox proportional hazard models and survival curves. The blood pressure subgroup with low systolic blood pressure $(<90 \mathrm{mmHg})$ and low diastolic blood pressure $(<60 \mathrm{mmHg})$ had the highest relative risk of mortality ( $\mathrm{HR}=2.34,95 \% \mathrm{CI}: 1.45-3.80)$, followed by those with normal systolic blood pressure but low diastolic blood pressure (HR=1.45, 95\% CI: 1.17-1.81) among those with cardiovascular conditions at baseline. For those without cardiovascular conditions at baseline, low blood pressure, either systolic or diastolic, was not related to mortality. Those with high levels of both systolic and diastolic blood pressure had a higher risk of mortality than those with both blood pressures normal but no other subgroups with low blood pressure differed from normal/normal in predicting mortality. Pulse pressure did not predict mortality. How high and low blood pressures are related to mortality needs to be examined by jointly looking at systolic and diastolic blood pressure.

Keywords: Systolic blood pressure, diastolic blood pressure, pulse pressure, mortality, Health and Retirement Study.
\end{abstract}

\section{Introduction}

It is accepted that abnormal blood pressure is related to mortality. Those who have out of range blood pressure have higher mortality than those within the normal range. High blood pressure (hypertension) and low blood pressure (hypotension) are defined as above or below certain cutoffs. Hypertension can be defined as either systolic blood pressure $>=140 \mathrm{mmHg}$ or diastolic blood pressure $>=90 \mathrm{mmHg}$; hypotension as either systolic blood pressure $<90 \mathrm{mmHg}$ or diastolic blood pressure $<60 \mathrm{mmHg}$. While those whose blood pressures are out of reference ranges, either lower or higher, are at higher risk of cardiovascular diseases and cardiovascular and all-cause mortality [1-5], more attention is generally given to hypertension at older ages partly because hypertension is much more prevalent among older people and because most treatment for hypertension is in response to high systolic blood pressure.
High blood pressure has been shown to cause serious health outcomes including mortality, aneurysm, stroke, chronic kidney disease, eye damage, heart attack, heart failure, peripheral artery disease and vascular dementia $[6,7]$. As much as hypertension is a critical indicator of health outcomes in old ages, hypotension is also a detrimental condition, related to higher mortality [1,3-5].

Corresponding Author: Jung Ki Kim, Davis School of Gerontology University of Southern California 3715 McClintock Avenue, Los Angeles, CA 90089-0191, USA Received date: October 13, 2020; Accepted date: October 20, 2020; Published date: October 21, 2020.

DOI: https://doi.org/10.31546/2633-7916.1009 
Systolic blood pressure increases with age due to the increasing stiffness of large arteries, long-term build-up of plaque and an increased incidence of cardiac and vascular disease $[8,9]$, and its increase at older ages is related to higher cardiovascular and all-cause mortality $[10,11]$. Some studies report that cardiovascular health may be better predicted by systolic blood pressure as people age while diastolic blood pressure may be a stronger predictor at younger ages [11].

While it is clear that both hypertension and hypotension based on systolic and diastolic blood pressures are important determinants of health outcomes at older ages, the importance of systolic and diastolic pressure and their relationship to mortality may differ when their effect is jointly viewed, which is not captured by conventional definitions of hypertension and hypotension. Some people may be low or high in both measures, while others may be high in one but low or normal in the other, and, thus, how combinations of the two blood pressure measures play a role in mortality needs to be examined.

Since systolic blood pressure tends to increase and diastolic blood pressure tends to decrease with increasing age, the difference between systolic and diastolic blood pressure, pulse pressure, adds an additional indicator to be examined in relating blood pressures and mortality at older ages. A widened pulse pressure may mean lower diastolic blood pressure or higher systolic blood pressure or both, all of them indicating arterial stiffness, atherosclerosis, or increased arterial stiffness [12].

Previous studies have examined the relative importance of systolic, diastolic and pulse pressure on mortality [12-18]. Pastor-Barriuso et al. examined the joint effect of systolic and diastolic blood pressure on mortality by examining how increasing systolic blood pressure for a fixed diastolic blood pressure and how increasing diastolic blood pressure for a fixed systolic blood pressure are related to relative risk of mortality [16]. They found that, among people aged 65 and over, increasing systolic blood pressure was related to a linear increase in mortality risk for all diastolic blood pressure levels, but for a fixed systolic blood pressure, increasing diastolic blood pressure was associated with decreased mortality risk for diastolic blood pressure below $80 \mathrm{mmHg}$ and increased mortality risk for diastolic blood pressure above $90 \mathrm{mmHg}$, showing a J-shaped relationship between diastolic blood pressure and mortality. Increased pulse pressure due to increasing systolic blood pressure was shown to be related to increased risk of mortality while increased pulse pressure due to decreasing diastolic blood pressure was related to increased risk only when diastolic blood pressure was above $90 \mathrm{mmHg}$; it was related to decreased risk when diastolic blood pressure was below $80 \mathrm{mmHg}$. While this study showed the combined effect of two blood pressure measures and pulse pressure on mortality, their blood pressure categories did not include separate low levels of systolic and diastolic blood pressure. Glynn et al. examined which combinations of three blood pressure measures best predict mortality among older people, and found that those with high systolic blood pressure and low diastolic blood pressure had higher risk of mortality and thus pulse pressure was the best single blood pressure measure for mortality prediction [13]. While they examined the relative importance of three blood pressure measures, their sample did not include low systolic blood pressure ranges but focused on high systolic blood pressure (but both low and high ranges of diastolic blood pressure). Although a number of previous efforts have been made to examine the joint effects of different blood pressure components, no studies that we have found have addressed blood pressures ranging from low to high in all three components of blood pressure.

We are looking at the relative importance or joint effect of systolic and diastolic blood pressure and pulse pressure on mortality, with both systolic and diastolic blood pressure levels ranging from low to high. While systolic and diastolic blood pressures are linearly related, it is not clear how levels of these blood pressure measures along with pulse pressure differentially predict mortality depending on the combination of their risk level or when considering other blood pressure measures. The differential changes of blood pressure components at older ages make it more critical to understand their joint effect on mortality.

\section{Materials and Methods Data and Study Participants}

Data for this study come from the 2006 to 2016 Health and Retirement Study (HRS). The HRS is a nationally representative survey of the U.S. population collected every two years since 1992. Blood pressure measures are examined every four years, with half of the sample first measured in 2006 and the other half in 2008, and they each have follow-up exams every four years after the first exam. The HRS has collected biomarkers from DBS on the same schedule. We used DBS assays from 2006/2008. 
Among the initial 13,125 participants who were ages 50 and over who agreed to the exam where blood pressure was collected (i.e., had a physical exam weight), 12,807 had systolic and diastolic blood pressure measures from either 2006 or 2008. Among these, 2,788 had missing information on antihypertensive medications $(\mathrm{N}=338), \quad$ cardiovascular conditions $(\mathrm{N}=319)$, body mass index $(\mathrm{N}=797)$, total cholesterol $(\mathrm{N}=1,789)$, glycated hemoglobin (HbA1c) $(\mathrm{N}=1,272)$ and death $(\mathrm{N}=190)$. Our final analytic sample is 10,336 with one additional case excluded based on our decision in the process of classifying blood pressure subgroups, which will be discussed in the measures section. Those who had missing information were similar to our analytic sample in terms of age, percent female, use of antihypertensive medications, BMI, lifetime pack years of smoking. Those with missing information were more likely to have lower total cholesterol $(194.9 \mathrm{mg} / \mathrm{dl}$ vs. $201.4 \mathrm{mg} / \mathrm{dl}$, $\mathrm{p}<.0001)$ and higher HbA1c (6.0\% vs. $5.9 \%$, p<.0001). Those excluded from the sample had a higher rate of death (29.4\%) compared to those in our final sample (27.3\%) ( $\mathrm{p}=.0312)$.

\section{Measures \\ Blood Pressure}

Systolic and diastolic blood pressures were measured three times, 45 seconds apart, on the respondent's left arm; we averaged the readings. Pulse pressure was calculated by subtracting diastolic blood pressure from systolic blood pressure. Systolic blood pressure was highly correlated with diastolic blood pressure $(\mathrm{r}=0.74, \mathrm{p}<.0001)$ and pulse pressure $(\mathrm{r}=0.84, \mathrm{p}<.0001)$ while the correlation coefficient between diastolic blood pressure and pulse pressure was lower $(\mathrm{r}=0.25$, $\mathrm{p}<.0001)$. We defined three levels of systolic blood pressure: $<90 \mathrm{mmHg}$ as low, $90-139 \mathrm{mmHg}$ as normal, and $>=140$ $\mathrm{mmHg}$ as high. Diastolic blood pressure was grouped into $<60$ $\mathrm{mmHg}$ as low, $60-89 \mathrm{mmHg}$ as normal, and $>=90 \mathrm{mmHg}$ as high. We cross-tabulated low, normal and high levels of systolic and diastolic blood pressures to produce subgroups of systolic/diastolic blood pressure levels. While there are 9 possible combinations of the two measures of blood pressure, there was no case of low systolic blood pressure and high diastolic blood pressure, and there was only one case of high systolic blood pressure and low diastolic blood pressure. Thus, we used 7 categories of systolic (SBP) and diastolic blood pressure (DBP) levels: (1) low/low, (2) low/normal, (3) normal/low, (4) normal/normal, (5) normal/high, (6) high/normal, and (7) high/high. For some analyses, we merged the first two subgroups, which both included blood pressure groups of low SBP and/or low DBP, in order to have enough cases to run the models for mortality separately by baseline cardiovascular conditions.
For pulse pressure, three categories of low $(<40$ $\mathrm{mmHg}$ ), normal (40-60 $\mathrm{mmHg})$, and high $(>60$ $\mathrm{mmHg}$ ) were designated.

\section{Mortality}

Mortality was tracked until 2016. Participants were followed to 2016 or until they died or exited from the survey for other reasons. Exposure time for those who died or exited between waves was assigned as 1 year or half the interval between interviews. Exposure times ranged from 1 to 10 years (average follow-up time of 7.8 years).

We examined all-cause mortality instead of cardiovascular mortality as risk trends for cardiovascular death and all-cause mortality by blood pressure are similar [16]. Total mortality had more cases and we wish to examine low blood pressure, which has relatively low frequency $(0.5 \%$ for low/low and $0.2 \%$ for low/normal, Table 1$)$.

\section{Covariates}

Sociodemographic information and health conditions at baseline were controlled. Age, gender, education, body mass index, total cholesterol and HbA1c level from DBS, whether taking antihypertensive medication, and lifetime smoking pack years were controlled. These characteristics were selected for their plausible associations with blood pressure and mortality. Analyses were run separately for those with and without baseline cardiovascular conditions defined as having any heart condition such as heart attack, coronary heart disease, angina, congestive heart failure and stroke. Baseline measures were from 2006 for those whose blood pressure was measured in 2006, and 2008 for those with 2008 blood pressure measures.

\section{Statistical Analysis}

First, we examined the relationship between the three components of blood pressure by age. Then, we showed descriptive statistics for the subgroups of systolic and diastolic blood pressure. We ran Cox proportional hazard models that predicted time to death to examine the association of different combinations of systolic/diastolic blood pressure levels and pulse pressure subgroups with all baseline covariates controlled. These models were run separately for those with and without cardiovascular conditions at baseline given that baseline cardiovascular condition may change the effect of blood pressure on mortality. 
Finally, we created adjusted survival curves for seven subgroups of systolic and diastolic blood pressures and for three pulse pressure subgroups. Since many covariates influence the relationship between blood pressure and mortality, we adjusted the Kaplan-Meier survival curves for the covariates: age, gender, education, total cholesterol, HbA1c, body mass index, smoking, and status of antihypertensive medication intake. To adjust survival curves, we assigned the mean of each covariate. In all analyses, we used sample weights to adjust for complex sampling design and nonresponse, and all analyses were conducted using SAS 9.4 .

\section{Results}

Almost two-thirds $(63.4 \%)$ had normal systolic and normal diastolic blood pressure while $14.7 \%$ had high/high and $0.5 \%$ had low/low (Table 1). About $2.9 \%$ had measured low systolic and/or diastolic blood pressure while $33.7 \%$ had measured high systolic and/or diastolic blood pressure. A little less than one fourth of the study population died by 2016. About a quarter of the sample $(25.8 \%)$ had a cardiovascular condition at baseline.

Blood pressure levels differed by age and the differences varied across the different components of blood pressure (Figure 1). The percent of those with high systolic blood pressure was double at ages $90+(45.9 \%)$ relative to ages 50-59 (22.4\%). Low systolic blood pressure was also higher at older ages: $0.8 \%$ at ages $50-59$ and $1.8 \%$ at ages $90+$. On the other hand, the percent with systolic blood pressure in the normal range was much smaller at older ages: that is, more than 3 out of 4 people $(77 \%)$ had normal systolic blood pressure among those aged 50-59, but only about half (53\%) of those aged $90+$ had normal systolic blood pressure. Diastolic blood pressure showed a different pattern by age. The percent with high diastolic blood pressure was somewhat lower at the oldest ages while the percent with low diastolic blood pressure was somewhat higher at older ages. The percent in the normal range was similar across age groups. Increased systolic blood pressure and decreased diastolic blood pressure at older ages led to a higher percent with high pulse pressure and a lower percent with low pulse pressure at older ages (high pulse pressure, $9.5 \%$ for ages $50-59$ and $52.7 \%$ for ages $90+$; low pulse pressure, $34.3 \%$ for ages $50-59$ and $2.6 \%$ for ages $90+$ ).

Next, we examined the means and variability of the blood pressure measures and mean pulse pressure by subgroup, and the percent of those who died by 2016 in those groups (Table 2). Comparing the two subgroups with low systolic blood pressure, mean systolic blood pressure was lower when diastolic blood pressure was low (85 vs. 87.1); comparing two high systolic blood pressure groups, mean systolic blood pressure was $10 \mathrm{mmHg}$ higher when diastolic blood pressure was high. Similarly, comparing two low diastolic blood pressure subgroups, mean diastolic blood pressure was lower when systolic blood pressure was low (55.2) than when systolic blood pressure was normal (56.4); among two high diastolic blood pressure subgroups, the mean of diastolic blood pressure with high systolic blood pressure was about $5 \mathrm{mmHg}$ higher than when systolic blood pressure was normal (98.2 vs 93.3). This means that, for example, even though people were classified as having normal systolic blood pressure, the mean of systolic blood pressure was higher when diastolic blood pressure was high. This is obvious by looking at pulse pressure. High pulse pressure was concentrated among those with high systolic pressure regardless of diastolic blood pressure level, and low pulse pressure was concentrated among those with low diastolic blood pressure regardless of systolic blood pressure. This indicates the complex dynamics among systolic and diastolic blood pressure, and pulse pressure that should be considered when examining the relationship between blood pressure and mortality.

Table 1: Description of the Sample, Weighted $(\mathrm{N}=10,336)$

\begin{tabular}{|l|l|}
\hline & Mean (SD) or Percent \\
\hline $\begin{array}{l}\text { \%Death by 2016 } \\
\text { combination }\end{array}$ & $22.5 \%$ \\
\hline Low/Low & \\
\hline Low/Normal & $0.5 \%$ \\
\hline Low/High & $0.2 \%$ \\
\hline Normal/Low & $\mathrm{NA}$ \\
\hline Normal/Normal & $2.2 \%$ \\
\hline Normal/High & $63.4 \%$ \\
\hline High/Low & $3.9 \%$ \\
\hline High/Normal & $\mathrm{NA}$ \\
\hline High/High & $15.1 \%$ \\
\hline$\%$ Pulse pressure combination & $14.7 \%$ \\
\hline Low & \\
\hline Normal & $21.5 \%$ \\
\hline High & $55.9 \%$ \\
\hline Baseline age & $22.6 \%$ \\
\hline$\%$ Female & $65.87(10.71)$ \\
\hline Baseline BMI & $54.1 \%$ \\
\hline Baseline total cholesterol & $29.32(6.36)$ \\
\hline Baseline HbA1c & $203.74(45.96)$ \\
\hline $\begin{array}{l}\text { \%Taking antihypertensive medication at } \\
\text { baseline }\end{array}$ & $5.82(1.06)$ \\
\hline Lifelong smoking pack years & $48.5 \%$ \\
\hline NA Notare & $17.77(21.82)$ \\
\hline
\end{tabular}

NA: Not available

SD: Standard Deviation 


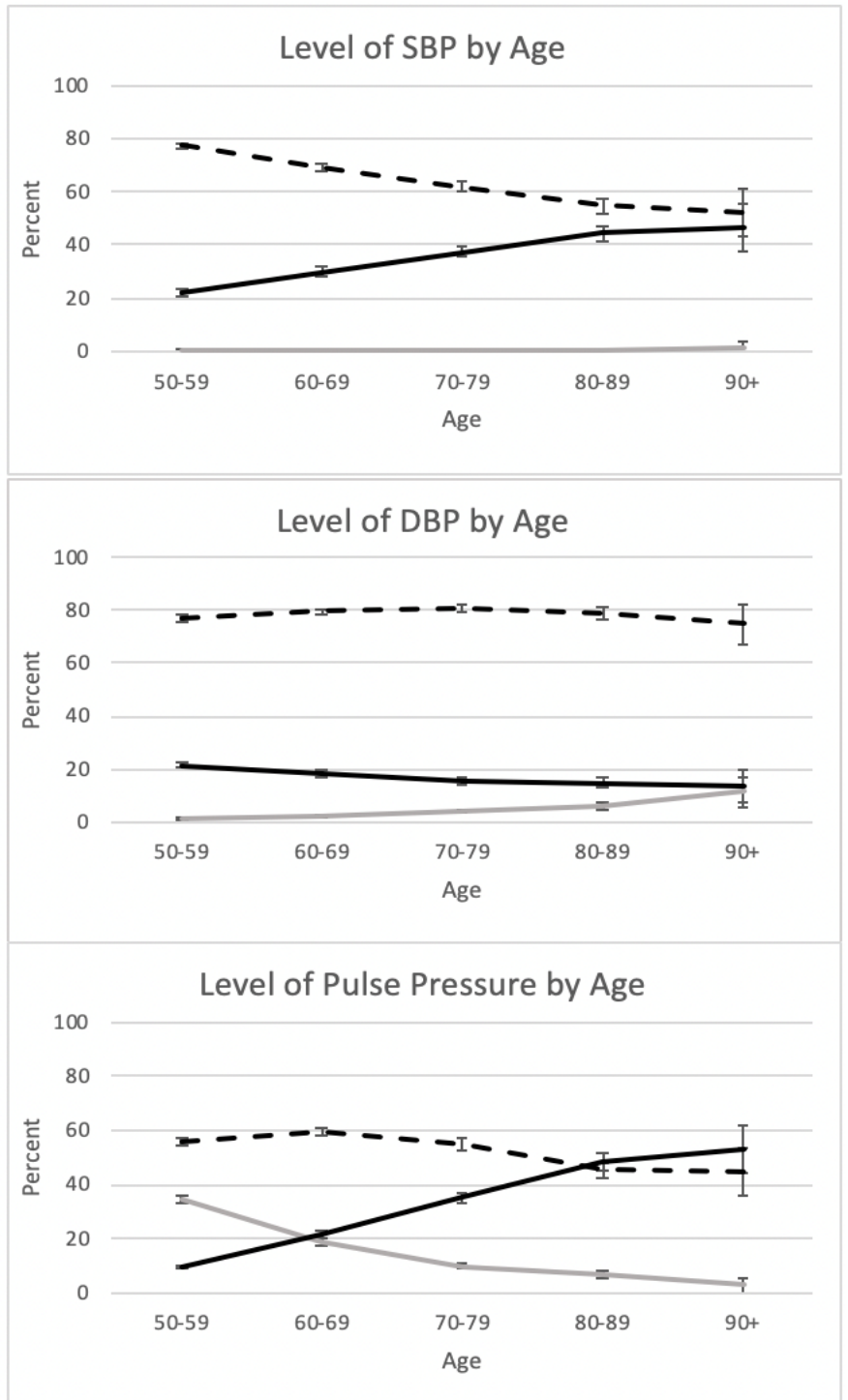

Figure 1: Systolic Blood Pressure (SBP), Diastolic Blood Pressure (DBP) and Pulse Pressure by Age

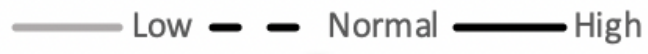

Also, those with normal systolic blood pressure but low diastolic blood pressure had the highest mortality rate (48\%) among the seven systolic/diastolic subgroups. While those with low diastolic blood pressure tended to have higher mortality rates, whether their systolic blood pressure was low or normal made about $20 \%$ difference in mortality rate $(28.8 \%$ vs. $48 \%)$ (Table 2). High systolic blood pressure was riskier for death when diastolic was normal rather than high. This suggests that pulse pressure might play a more critical role in determining mortality.

Next, we examined the relative importance of joint indicators of systolic and diastolic blood pressure and pulse pressure on mortality with controls and separately for those with and without cardiovascular conditions.
Hazard ratios for each blood pressure and pulse pressure subgroup are shown relative to normal/normal blood pressure and normal pulse pressure subgroups (Table 3), and the associated survival curves are presented in Figure 2.

In the model for those with cardiovascular conditions at baseline, those with low/low or low/normal levels of systolic and diastolic blood pressure were 2.34 times more likely to experience mortality at any time after $2006 / 2008$ to 2016 than those with normal/normal (95\% CI: 1.45-3.80, $\mathrm{p}=<.001$ ); those with normal systolic and low diastolic blood pressure had 1.45 times higher risk of mortality than those with normal/normal blood pressures (95\% CI: 1.17-1.81, $\mathrm{p}=<.001)$. No significant difference in mortality risk was found for normal/high $(\mathrm{HR}=1.20,95 \% \mathrm{CI}$ : 0.73 1.98, $\mathrm{p}=.466)$, high/normal ( $\mathrm{HR}=1.11,95 \% \mathrm{CI}: 0.97$ $1.27, \mathrm{p}=.115)$ and high/high $(\mathrm{HR}=1.03$, 95\% CI: 0.861.23, $\mathrm{p}=.781$ ) compared to normal/normal. In the model with no cardiovascular conditions at baseline, a significant increase in relative risk of mortality was observed between high/high and normal/normal, but no other subgroups. We also examined the effect of pulse pressure on mortality. Our models showed that pulse pressure did not predict mortality risk. Regardless of cardiovascular conditions at baseline, low, normal and high pulse pressure groups did not differ in their risk of mortality when all proposed covariates were controlled.

Among those with cardiovascular conditions at baseline, the probability of survival was highest for the normal/normal subgroup, followed by those with high systolic and high diastolic blood pressure. Those whose systolic blood pressure was high and diastolic blood pressure was normal and those with normal/high levels survived shorter than high/high subgroup. Those with low values of either systolic or diastolic blood pressure had shorter survival than other subgroups. Particularly, those with low systolic blood pressure survived the shortest time. While the low/low or low/normal group was least likely to survive among those with cardiovascular conditions at baseline, the difference among these groups for those without baseline conditions was not shown. The survival curves of the three pulse pressure subgroups were close to each other, showing no significant difference in survival particularly among those without baseline cardiovascular conditions. 


\section{Jung Ki Kim}

Table 2: Means of Blood Pressure Subgroups

\begin{tabular}{|c|c|c|c|c|c|c|c|c|c|c|c|}
\hline & & \multicolumn{3}{|c|}{ SBP/DBP } & & & \multicolumn{4}{|c|}{ Pulse Pressure } & \multirow[t]{2}{*}{$\begin{array}{l}\text { \%Died by } \\
2016\end{array}$} \\
\hline & & Mean & Std Dev & Min & Max & Mean & Std Dev & Low & Normal & High & \\
\hline Low/Low & $\begin{array}{l}\text { SBP } \\
\text { DBP }\end{array}$ & $\begin{array}{l}85.0 \\
55.2 \\
\end{array}$ & $\begin{array}{l}5.1 \\
4.2\end{array}$ & $\begin{array}{l}68.5 \\
41.7\end{array}$ & $\begin{array}{l}89.7 \\
59.7 \\
\end{array}$ & 29.8 & 5.4 & $97.8 \%$ & $2.2 \%$ & $0.0 \%$ & $28.8 \%$ \\
\hline Low/Normal & $\begin{array}{l}\text { SBP } \\
\text { DBP } \\
\end{array}$ & $\begin{array}{l}87.1 \\
62.6 \\
\end{array}$ & $\begin{array}{l}2.4 \\
2.8 \\
\end{array}$ & $\begin{array}{l}81.7 \\
60.0 \\
\end{array}$ & $\begin{array}{l}89.7 \\
67.5 \\
\end{array}$ & 24.6 & 3.7 & $100.0 \%$ & $0.0 \%$ & $0.0 \%$ & $22.5 \%$ \\
\hline Normal/Low & $\begin{array}{l}\text { SBP } \\
\text { DBP }\end{array}$ & $\begin{array}{c}106.2 \\
56.4\end{array}$ & $\begin{array}{c}11.3 \\
2.8 \\
\end{array}$ & $\begin{array}{l}90.0 \\
45.3 \\
\end{array}$ & $\begin{array}{c}139.7 \\
59.7 \\
\end{array}$ & 49.8 & 10.8 & $20.8 \%$ & $60.3 \%$ & $18.9 \%$ & $48.0 \%$ \\
\hline Normal/Normal & $\begin{array}{l}\text { SBP } \\
\text { DBP }\end{array}$ & $\begin{array}{c}120.9 \\
75.8\end{array}$ & $\begin{array}{c}11.9 \\
8.0\end{array}$ & $\begin{array}{l}90.0 \\
60.0\end{array}$ & $\begin{array}{c}139.7 \\
89.7\end{array}$ & 45.1 & 10.0 & $29.6 \%$ & $64.8 \%$ & $5.6 \%$ & $18.6 \%$ \\
\hline Normal/High & $\begin{array}{l}\text { SBP } \\
\text { DBP }\end{array}$ & $\begin{array}{l}133.6 \\
93.3 \\
\end{array}$ & $\begin{array}{l}6.3 \\
3.9 \\
\end{array}$ & \begin{tabular}{|l}
99.0 \\
90.0 \\
\end{tabular} & $\begin{array}{l}139.7 \\
104.7 \\
\end{array}$ & 40.3 & 6.9 & $41.8 \%$ & $58.2 \%$ & $0.0 \%$ & $14.0 \%$ \\
\hline High/Normal & $\begin{array}{l}\text { SBP } \\
\text { DBP } \\
\end{array}$ & $\begin{array}{c}150.3 \\
82.0\end{array}$ & $\begin{array}{c}10.0 \\
5.9 \\
\end{array}$ & $\begin{array}{c}140.0 \\
60.0\end{array}$ & $\begin{array}{c}205.7 \\
89.7 \\
\end{array}$ & 68.4 & 10.9 & $0.0 \%$ & $23.9 \%$ & $76.1 \%$ & $36.6 \%$ \\
\hline High/High & $\begin{array}{l}\text { SBP } \\
\text { DBP }\end{array}$ & $\begin{array}{c}160.3 \\
98.2\end{array}$ & $\begin{array}{c}17.3 \\
8.1\end{array}$ & $\begin{array}{c}140.0 \\
90.0\end{array}$ & $\begin{array}{l}227.3 \\
145.0\end{array}$ & 62.1 & 14.8 & $0.8 \%$ & $51.4 \%$ & $47.8 \%$ & $22.9 \%$ \\
\hline
\end{tabular}

SBP: Systolic Blood Pressure

DBP: Diastolic Blood Pressure

(a) Systolic/Diastolic Blood Pressure Subgroups

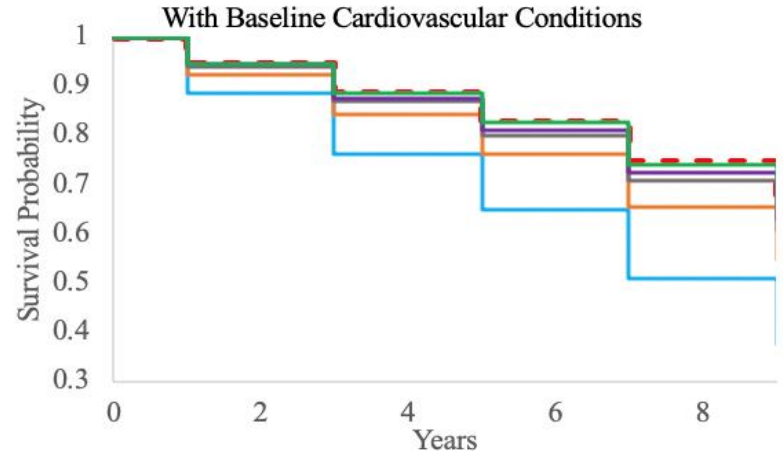

(b) Pulse Pressure Subgroups

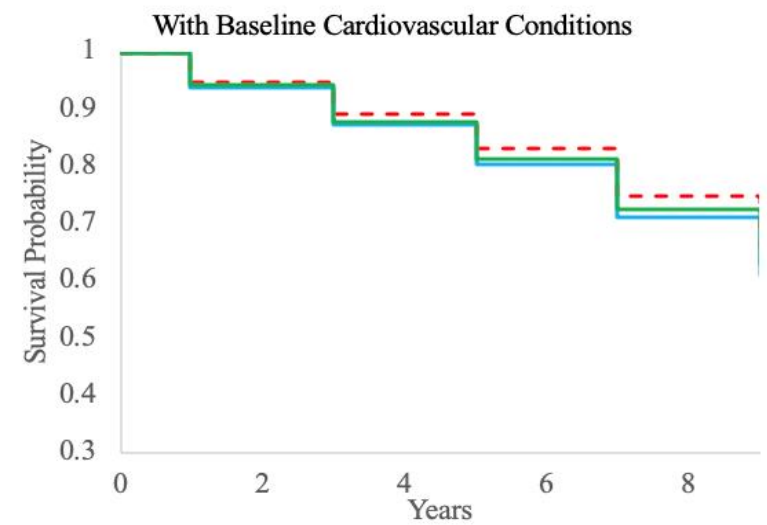

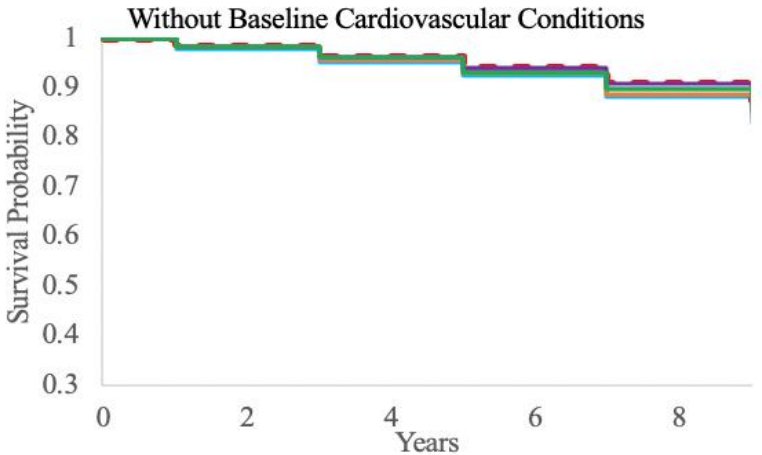

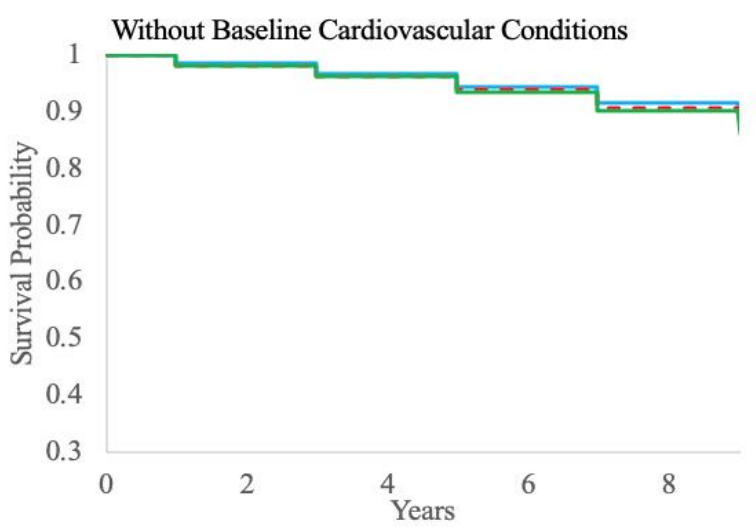

Figure 2: Survival Curve by Blood Pressure Categories

a. Systolic/ Diastolic Blood Pressure Subgroups

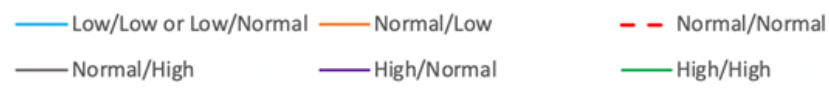

b. Pulse Pressure Subgroups

$$
\text { L Low - - - Normal _-High }
$$


Jung Ki Kim

Table 3: Relative Risk of Death by Blood Pressure Components (N=10,336)

\begin{tabular}{|c|c|c|c|c|c|c|c|c|c|c|c|c|}
\hline & \multicolumn{5}{|c|}{ SBP/DBP } & \multicolumn{7}{|c|}{ Pulse Pressure } \\
\hline & \multicolumn{3}{|c|}{$\begin{array}{l}\text { With baseline cardiovascular } \\
\text { conditions }(\mathrm{N}=2,973)\end{array}$} & \multicolumn{2}{|c|}{$\begin{array}{c}\text { Without baseline cardiovascular } \\
\text { conditions }(\mathrm{N}=7,363)\end{array}$} & \multirow[b]{2}{*}{$\dot{p}$} & \multicolumn{2}{|c|}{$\begin{array}{l}\text { With baseline cardiovascular } \\
\text { conditions }(N=2,973)\end{array}$} & \multicolumn{4}{|c|}{$\begin{array}{c}\text { Without baseline cardiovascular } \\
\text { conditions }(\mathrm{N}=7,363)\end{array}$} \\
\hline & HR & $95 \% \mathrm{CI}$ & $\dot{p}$ & HR & $95 \% \mathrm{CI}$ & & HR & $95 \% \mathrm{CI}$ & $\dot{p}$ & $\mathrm{HR}$ & $95 \% \mathrm{CI}$ & $\mathrm{p}$ \\
\hline \multicolumn{13}{|l|}{$\begin{array}{l}\text { Blood Pressure Groups } \\
\text { (SBP/DBP) }\end{array}$} \\
\hline \multicolumn{13}{|l|}{ Normal/Normal (reference) } \\
\hline Low/Low or Low/Normal & 2.34 & $1.45,3.80$ & $<.001$ & 1.41 & $0.70,2.84$ & 0.335 & & & & & & \\
\hline Normal/Low & 1.45 & $1.17,1.81$ & $<.001$ & 1.30 & $0.97,1.75$ & 0.080 & & & & & & \\
\hline Normal/High & 1.20 & $0.73,1.98$ & 0.466 & 1.19 & $0.83,1.70$ & 0.338 & & & & & & \\
\hline High/Normal & 1.11 & $0.97,1.27$ & 0.115 & 1.09 & $0.96,1.23$ & 0.207 & & & & & & \\
\hline High/High & 1.03 & $0.86,1.23$ & 0.781 & 1.21 & $1.06,1.40$ & 0.007 & & & & & & \\
\hline \multicolumn{13}{|l|}{ Pulse pressure groups } \\
\hline \multicolumn{13}{|l|}{ Normal (reference) } \\
\hline Low & & & & & & & 1.19 & $0.98,1.43$ & 0.074 & 0.94 & $0.79,1.12$ & 0.473 \\
\hline High & & & & & & & 1.12 & $0.99,1.26$ & 0.074 & 1.08 & $0.97,1.21$ & 0.175 \\
\hline Baseline age & 1.08 & $1.07,1.09$ & $<.001$ & 1.10 & $1.09,1.11$ & $<, 001$ & 1.08 & $1.08,1.09$ & $<.001$ & 1.10 & $1.09,1.11$ & $<, 001$ \\
\hline Female & 0.93 & $0.82,1.05$ & 0.227 & 0.75 & $0.67,0.83$ & $<, 001$ & 0.91 & $0.81,1.03$ & 0.129 & 0.76 & $0.68,0.84$ & $<.001$ \\
\hline Baseline BMI & 0.99 & $0.98,1.00$ & 0.190 & 0.98 & $0.97,0.99$ & $<.001$ & 0.99 & $0.98,1.00$ & 0.151 & 0.98 & $0.97,0.99$ & $<.001$ \\
\hline Baseline total cholesterol & 1.00 & $1.00,1.00$ & 0.608 & 1.00 & $1.00,1.00$ & 0.512 & 1.00 & $1.00,1.00$ & 0.396 & 1.00 & $1.00,1.00$ & 0.547 \\
\hline Baseline $\mathrm{HbAlc}$ & 1.16 & $1.12,1.23$ & $<.001$ & 1.19 & $1.13,1.24$ & $<.001$ & 1.17 & $1.11,1.23$ & $<.001$ & 1.18 & $1.13,1.24$ & $<, 001$ \\
\hline $\begin{array}{l}\text { Taking antihypertensive } \\
\text { medication at baseline }\end{array}$ & 1.19 & $1.04,1.35$ & 0.010 & 1.08 & $0.97,1.20$ & 0.162 & 1.17 & $1.03,1.33$ & 0.020 & 1.07 & $0.96,1.18$ & 0.233 \\
\hline Lifelong smoking pack years & 1.01 & $1.01,1.01$ & $<.001$ & 1.01 & $1.01,1.02$ & $<, 001$ & 1.01 & $1.01,1.01$ & $<.001$ & 1.01 & $1.01,1.02$ & $<.001$ \\
\hline
\end{tabular}

\section{Conclusion}

Our study found that the most common form of high blood pressure, isolated systolic hypertension (high systolic but normal diastolic blood pressure), did not differ from normal/normal blood pressure measures in predicting mortality while isolated diastolic hypotension (normal systolic but low diastolic blood pressure) was related to higher mortality. This is similar to other studies' findings on isolated diastolic hypotension as a risk factor for cardiovascular health outcomes [19].

After adjusting for significant covariates, survival time for the low systolic blood pressure and low diastolic blood pressure subgroup was shortest. The difference in the survival probabilities between the low/low or low/normal subgroup and other subgroups was more pronounced in survival curves among those with baseline cardiovascular conditions than among those without conditions. Low systolic or diastolic blood pressure in any combination of systolic and diastolic blood pressure did not predict mortality among those without baseline cardiovascular conditions. This finding coincides with previous findings that those with low blood pressure had higher mortality rate because of poor health, particularly cardiovascular, rather than low blood pressure causing high mortality risk [7,20-22]. The effect of systolic and diastolic blood pressure and that of hypertension and hypotension on mortality need to be examined and monitored differently for those with and without underlying cardiovascular conditions.

We also showed that high blood pressure was not as predictive of mortality as low blood pressure among those with cardio- -vascular conditions at baseline. This finding is consistent with previous findings that hypertension may be a less important risk factor for mortality for very old people [21], and increased systolic blood pressure may not be linearly associated with increased mortality risk [23]. However, the previous finding that higher systolic and diastolic blood pressure predicted linear increases in mortality and low blood pressure in older ages might be related to better survival after excluding short-term death [2] was not supported by our additional analysis. We examined the same blood pressure and death relationship only considering death from 2 to 10 years after blood pressure measurement in order to eliminate the associated confounding effect of frailty and comorbidity with low blood pressure near death, and found that low blood pressure still predicted higher mortality risk.

The high systolic and high diastolic blood pressure subgroup exhibited a significantly higher relative risk for mortality only among those without baseline cardiovascular conditions. Those high in one measure but normal in the other did not differ in mortality from other subgroups. On the other hand, those with low levels of blood pressure, either systolic or diastolic, had higher mortality while those with low/low had the worst survival. This implies that using a measure of hypertension based on having either high systolic or diastolic blood pressure may not provide an accurate picture of the association of blood pressure to mortality. 
In contrast to some previous studies $[11,13,17]$, our study didn't find that level of pulse pressure predicted mortality. How narrow or widened pulse pressure affected mortality depended on the levels of systolic and diastolic blood pressure instead of pulse pressure per se. Given that our findings showed that systolic and diastolic blood pressure need to be jointly viewed in relation to mortality, it makes sense that pulse pressure which is highly correlated with systolic blood pressure, but not with diastolic blood pressure, did not predict mortality.

Mixed findings from previous studies on the relative importance of hypertension vs hypotension and systolic blood pressure vs diastolic blood pressure, and pulse pressure indicate the complexity of the links between blood pressure and mortality. It is hard to summarize with a simple statement which blood pressure component, or hypertension or hypotension, predicts mortality better. Our study also suggested a complex picture of how blood pressure components jointly relate to mortality risk.

Acknowledgements: None.

Funding: This study is funded by the National Institute on Aging (P30 AG17265).

Conflicts of Interest: The authors declare no conflict of interest.

\section{References:}

1. Amnuaypattanapon K, Khansompop S. Characteristics and factors associated with the mortality of hypotensive patients attending the emergency department. J Clin Med Res. 2018;10:576-581.

2. Glynn R, Field TS, Rosner B, et al. Evidence for a positive linear relation between blood pressure and mortality in elderly people. Lancet. 1995;345:825-829.

3. Holler JG, Bech CN, Henriksen DP, et al. Nontraumatic hypotension and shock in the emergency department and the prehospital setting, prevalence, etiology, and mortality: a systematic review. PLoS One. 2015;10:e0119331.

4. Imai Y, Ohkubo T, Sakuma M, et al. Predictive power of screening blood pressure, ambulatory blood pressure and blood pressure measured at home for overall and cardiovascular mortality: a prospective observation in a cohort from Ohasama, northern Japan. Blood Press Monit. 1996;1:251-254.

5. Masaki KH, Schatz IJ, Burchfiel CM, Sharp DS, et al. Orthostatic hypotension predicts mortality in elderly men. The Honolulu Heart Program. Circulation. 1988;98:22902295.
6. High blood pressure. National Heart, Lung, and Blood Institute. [internet]. Accessed July 25, 2020.

7. Kannel W, D'Agostino R, Silbershatz H. Blood pressure and cardiovascular morbidity and mortality rates in the elderly. Am Heart J. 1997; 134:758-763.

8. American Heart Association 2020. Understanding Blood Pressure Readings [internet]. Accessed July 20, 2020.

9. Blood Pressure UK 2008. Blood pressure symptoms, causes, medicines and lifestyle [internet]. Accessed July 20, 2020.

10. Bundy J, Li C, Stuchlik P, et al. Systolic blood pressure reduction and risk of cardiovascular disease and mortality. A systematic review and network meta-analysis. JAMA Cardiol. 2017; 2:775-781.

11. Franklin SS, Larson MG, Khan SA, et al. Does the relation of blood pressure to coronary heart disease risk change with aging? The Framingham Heart Study. Circulation. 2001;103:1245-1249.

12. Oh HJ, Lee S, Lee E, et al. Association of blood pressure components with mortality and cardiovascular events in prehypertensive individuals: a nationwide population-based cohort study. Ann Med 2018; 50: 443-452.

13. Glynn R, Chae C, Guralnik J, et al. Pulse pressure and mortality in older people. Arch Intern Med. 2000; 160: 2765-2772.

14. Lee TC, Cavalcanti RB, McDonald EG, et al. Diastolic hypotension may attenuate benefits from intensive systolic targets: secondary analysis of a randomized controlled trial. Am J Med. 2018;131:1228-1233.

15. Mancia G, De Backer G, Dominiczak A, et al. 2007 Guidelines for the management of arterial hypertension The Task Force for the Management of Arterial Hypertension of the European Society of Hypertension (ESH) and of the European Society of Cardiology (ESC). Eur Heart J. 2007;28:1462-1536.

16. Pastor-Barriuso R, Banegas JR, Damián J, Appel LJ, Guallar E. Systolic blood pressure, diastolic blood pressure, and pulse pressure: an evaluation of their joint effect on mortality. Ann Intern Med. 2003; 139:731-739.

17. Thomas F, et al. Cardiovascular risk as defined in the 2003 European blood pressure classification: the assessment of an additional predictive value of pulse pressure on mortality. J Hypertens. 2008:26:1072-1077. 
18. Tsimploulis A, Sheriff HM, Lam PH, et al. Systolicdiastolic hypertension versus isolated systolic hypertension and incident heart failure in older adults: Insights from the Cardiovascular Health Study. Int J Cardiol. 2017;235:1116.

19. Guichard J, Desal R, Ahmed MI, et al. Isolated diastolic hypotension and incident heart failure in older adults. Hypertension. 2011;58:895-901.

20. Boutitie F, Gueyffier F, Pocock S, et al. J-shaped relationship between blood pressure and mortality in hypertensive patients: New insights from a meta-analysis of individual-patient data. Ann Intern Med. 2002;136:438448.

21. Bulpitt CJ, Fletcher AE. Prognostic significance of blood pressure in the very old. Implications for the treatment decisions. Drugs Aging. 1994;5:184-191.

22. Langer RD, Ganiats TG, Barrett-Conner E. Paradoxical survival of elderly in men with high blood pressure. BMJ. 1989;298:1356-1358.

23. Port S, Demer L, Jennrich R, et al. Systolic blood pressure and mortality. Lancet. 2000;355:175-180. 